\title{
Epithelial and Mesothelial Basement Membranes in Diabetic Patients and Dogs
}

\author{
R. L. Engerman and P.J. Colquhoun \\ Department of Ophthalmology, University of Wisconsin, Madison, Wisconsin, USA
}

\begin{abstract}
Summary. The influence of diabetes on the thickness of basement membrane of the ciliary epithelium, lens epithelium, and corneal mesothelium (endothelium) was investigated post mortem in eyes from 27 human subjects and 30 dogs. Ten of the human subjects were diabetic, 10 were non-diabetic matched for age and sex, and seven were non-diabetic subjects who had been diagnosed as hypertensive $2-8$ years before death. The dogs comprised three prospectively identified groups: 10 were alloxan diabetic for 5 years and kept in poor glucose control, 10 were alloxan diabetic for 5 years and kept in good glucose control, and 10 were non-diabetic animals. Basement membranes at the three sites measured appeared to be normal in the seven non-diabetic hypertensive subjects. Basement membrane of the ciliary epithelium was found to be significantly thicker than normal both in diabetic human sub-
\end{abstract}

jects and in poorly controlled diabetic dogs. The thickness of the anterior lens capsule was significantly greater in poorly controlled diabetic dogs than in non-diabetic dogs, and showed a significant positive correlation with duration of diabetes in human subjects. Better glucose control in diabetic dogs resulted in significant inhibition of the epithelial basement membrane thickening. The basement membrane of the corneal mesothelium failed to thicken with diabetes both in human subjects and in animals.

Key words: Basement membrane, epithelium, mesothelium, diabetes mellitus, alloxan diabetes, diabetic control, dog, man, hypertension, eye, cornea, Descemet's membrane, ciliary process, lens.
Thickening of the basement membrane of blood vessels is one of the most common morphological abnormalities observed in diabetes mellitus. The thickening may be apparent within one or two years after onset of clinically recognizable diabetes [1], and occurs not only in man, but also in various animal models of diabetes. Studies in experimentally diabetic animals have indicated that the basement membrane of the blood vessel may thicken as a result of inadequate treatment of the diabetes, and fails to thicken if the diabetes is carefully controlled with insulin $[2,3]$. The mechanism by which such changes occur in blood vessels remains poorly understood.

Other basement membranes have also been reported to be abnormally thick in diabetic patients, such as epithelial basement membrane in the ciliary processes of the eye [4], and in some other body regions $[5,6]$. The basement membrane of the ciliary epithelium is commonly several-fold thicker than normal in autopsy eyes from diabetic patients, the increase often being readily evident by light microscopy. In the present study we have compared the influence of diabetes on the thick- ness of three non-vascular basement membranes in the eyes of man and dogs, and have sought to learn whether or not such basement membranes are affected by the degree of control of diabetes.

\section{Materials and Methods}

\section{Animals}

Healthy young adult dogs of mixed breeds were made diabetic by IV administration of alloxan and were assigned at random to be controlled either intentionally poorly or intentionally well with insulin. Details of our procedures have been published previously [7]. Animals in the prospective poor control group were allowed food ad libitum and given too little insulin to prevent chronic hyperglycaemia and glycosuria. Animals in the prospective good control group were given insulin and food twice daily in a manner intended to minimize hyperglycaemia and glycosuria. To achieve good control, food of a given animal was restricted to an amount that would be eaten promptly but sufficient to maintain normal body weight, and an insulin dose was carefully selected each morning and evening according to that animal's history. The insulin was an intermediate acting type, usually isophane insulin suspension (NPH, Lilly), alone or supplemented with soluble (regular) insulin (Iletin, Lilly). After 5 years of diabetes, 
Table 1. Thickness of epithelial and mesothelial basement membrane in diabetic patients

\begin{tabular}{|c|c|c|c|c|c|c|}
\hline & \multirow[t]{2}{*}{$\begin{array}{l}\text { Age } \\
\text { (years) }\end{array}$} & \multirow{2}{*}{$\begin{array}{l}\text { Diabetes } \\
\text { duration } \\
\text { (years) }\end{array}$} & \multicolumn{2}{|c|}{$\begin{array}{l}\text { Ciliary process } \\
\text { basement membrane }(\mu \mathrm{m})\end{array}$} & \multirow{2}{*}{$\begin{array}{l}\text { Lens } \\
\text { capsule } \\
(\mu \mathrm{m})\end{array}$} & \multirow{2}{*}{$\begin{array}{l}\text { Descemet's } \\
\text { membrane } \\
(\mu \mathrm{m})\end{array}$} \\
\hline & & & Random & Minimum & & \\
\hline \multicolumn{7}{|l|}{ Non-diabetic subjects } \\
\hline Hypertensive $(n=7)$ & $44.3 \pm 8$ & 0 & $4.08 \pm 0.7$ & $2.75 \pm 0.7$ & \multirow{2}{*}{$\begin{array}{l}11.2 \pm 3.3 \\
(n=5) \\
11.0 \pm 2.8\end{array}$} & \multirow{2}{*}{$\begin{array}{l}6.4 \pm 1.5 \\
(n=3) \\
6.5 \pm 1.0\end{array}$} \\
\hline Non-hypertensive $(n=10)$ & $44.7 \pm 8$ & 0 & $3.40 \pm 0.7$ & $2.17 \pm 0.4$ & & \\
\hline Diabetic patients $(n=10)$ & $44.5 \pm 9$ & $21.1 \pm 12$ & $10.22 \pm 3.2$ & $6.51 \pm 2.3$ & $\begin{array}{l}13.6 \pm 3.2 \\
(n=9)\end{array}$ & $5.8 \pm 1.2$ \\
\hline
\end{tabular}

Results expressed as mean $\pm \mathrm{SD}$

10 animals of each group were killed for comparison with 10 nondiabetic animals that had been housed in adjacent cages for periods of 3-6 years.

At necropsy the eyes were fixed in formalin, and the anterior segment was divided in a mid-saggital plane before being imbedded in paraffin. Saggital sections were cut at $6 \mu \mathrm{m}$ and stained with haematoxylin and a periodic acid-Schiff reaction. Representative sections were bleached with chlorine water to decolourize pigmented cells before staining, the section on the microscope slide having been coated beforehand with a thin layer of dilute celloidin to prevent loss of sections during bleaching.

\section{Patients}

Autopsy eyes from a total of 27 subjects were selected for study from the pathology archives of our department. Ten of these were diabetic subjects chosen so that they were 25-55 years of age at death, had been diabetic $>6$ years, were insulin-treated, and afforded eye sections physically suitable for analysis. Diabetic nephropathy had been diagnosed in three of the patients, and retinopathy in seven. The mean age at onset of diabetes was 23 years (range 10-41 years). Five of the diabetic patients had shown some evidence of vascular hypertension (i. e. systolic pressure $\geqq 160$ and/or diastolic $\geqq 90 \mathrm{mmHg}$ ). The duration of diabetes in these five patients was $27 \pm 12$ years (mean \pm SD), in contrast to $15 \pm 10$ years in the remaining five diabetic patients. In view of the near twofold difference in duration of diabetes between these two subgroups, comparison of their basement membranes was deemed inappropriate. The mean ( \pm SD) duration of diabetes for the total of 10 patients was $21 \pm 12$ years.

Ten subjects believed to be free of diabetes, hypertension, and ocular disease before death were matched with the diabetic patients for age and sex. We also studied tissue from seven non-diabetic patients of similar ages who had been diagnosed as hypertensive $2-8$ years before death. Blood pressure data were limited, but the group mean $( \pm$ SD) was $120 \pm 16 / 77 \pm 5 \mathrm{mmHg}$ for non-diabetic non-hypertensive subjects, $197 \pm 43 / 103 \pm 13 \mathrm{mmHg}$ for non-diabetic hypertensive subjects, $133 \pm 9 / 81 \pm 3 \mathrm{mmHg}$ for diabetic non-hypertensive patients, and $162 \pm 11 / 94 \pm 11 \mathrm{mmHg}$ for diabetic hypertensive patients.

Basement membranes were measured in sections stained like those of the dogs, and obtained from formalin-fixed eyes that had been imbedded in paraffin and cut at a thickness of $8 \mu \mathrm{m}$ in a midsaggital plane.

\section{Measurement of Basement Membranes}

A light microscope equipped with a calibrated ocular micrometer was used to measure the thickness of basement membrane. Measurements were made on tissue sections coded and masked so as to minimize observer bias. Measurements of the epithelial basement membrane were made under oil immersion $(\times 1250)$ and excluded those sites at which the epithelium seemingly had been cut tangentially or in which pigment was bleached inadequately or otherwise impaired the visibility of the basement membrane. In each subject, the ciliary basement membrane was measured at 12 points selected at random (using a table of random numbers and a numbered grid in a microscope ocular). In addition, the minimum thickness of this basement membrane also was estimated from nine measurements judged to represent the thinnest regions. Descemet's membrane and the anterior capsule of the lens were measured at a point along the optic axis of the eye (i.e., in the central cornea or lens); sections showing damage at this point were excluded from the analysis. Data were analyzed statistically by Student's t-test, and also by analysis of variance in the case of the ciliary basement membrane.

\section{Results}

Among non-diabetic subjects, a diagnosis of vascular hypertension was not associated with any apparent abnormality in the basement membranes examined (Table 1).

The basement membrane of the ciliary epithelium was significantly thicker in diabetic subjects than in either the hypertensive or the normotensive non-diabetic subjects $(p<0.01)$, regardless of whether the thickness was measured at random sites or at the thinnest portions of the membrane (Table 1). This thickening in diabetes amounted to a mean width of nearly twice normal, and although the thickening seemed to involve the ciliary process uniformly, it diminished quite abruptly at the root of the iris.

The anterior capsule of the lens (basement membrane of the lens epithelium) tended to be thicker in diabetic than in normal subjects (NS). The capsule thickness showed a significant positive correlation with the duration of diabetes $(r=0.67, p<0.05)$. This was not due simply to aging, since in non-diabetic subjects there was little correlation between capsule thickness and age $(r=0.15)$.

Descemet's membrane (the basement membrane of the corneal mesothelium) was clearly no thicker than normal in the diabetic subjects. The mean thickness in diabetic patients $(5.8 \mu \mathrm{m})$ tended to be less than in the non-diabetic subjects $(6.5 \mu \mathrm{m}, \mathrm{NS})$.

\section{Animals}

The basement membrane of the ciliary epithelium in normal adult dogs is only about one-tenth as thick as in 
Table 2. Influence of diabetic control on thickness of epithelial and mesothelial basement membrane in dogs alloxan-diabetic for 5 years

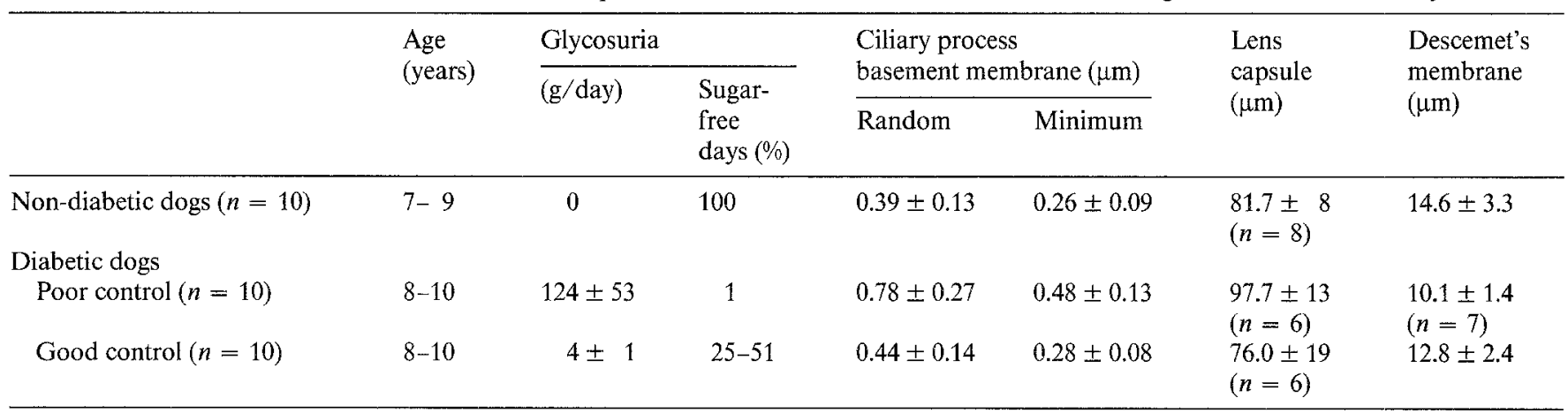

Results expressed as mean \pm SD

man, in contrast to the basement membrane of the lens epithelium and Descemet's membrane, each of which is appreciably thicker in dogs than in man (Table 2). The basement membrane of the ciliary epithelium in dogs was usually under $1 \mu \mathrm{m}$ thick, and was therefore difficult to measure, but significant thickening was nevertheless found to occur as a result of diabetes $(p<0.01$ when comparing either random or minimum measurements in poorly controlled diabetic dogs with those in normal dogs).

The anterior capsule of the lens (basement membrane of the lens epithelium) also was abnormally thick in poorly controlled diabetic dogs $(p<0.05)$. Lens opacities developed in all animals of the poorly controlled diabetic group, but were absent at autopsy in the well controlled diabetic group and in non-diabetic animals.

In contrast to the basement membrane thickening observed in the ciliary processes and lens, Descemet's membrane showed no thickening at all after 5 years of diabetes. Descemet's membrane was in fact thinner in the chronically glycosuric group of dogs than in either the normal $(p<0.01)$ or the well controlled group $(p<$ 0.05 ). The thickness observed in the well controlled group did not differ significantly from normal.

The thickening of epithelial basement membrane in the diabetic animals was found to be significantly inhibited as a result of better treatment of the diabetes $(p<$ 0.05 when comparing either the lens or ciliary processes of the poorly controlled and the well controlled groups, Table 2). Indeed, the basement membrane of the lens and ciliary processes remained no wider than normal in the well controlled diabetic group, in which hyperglycaemia, glycosuria, and other metabolic sequelae of deficient insulin activity had been suppressed [7] by twicedaily injections of commercial insulin.

\section{Discussion}

Basement membrane thickening has been demonstrated previously in diabetes, not only in the microvasculature of many tissues but also in basement membranes of the
Schwann cell [8], epineurium [9], and epithelia of lung alveoli [6], renal tubules [6], cornea [10], and ciliary processes [4]. Such thickening is said to occur also at a number of other sites, for example at the epithelium of testicular canaliculi, Bowman's capsule, and sweat glands of skin. In skin, the basement membrane of the epidermis reportedly may retain its normal thickness during diabetes, but in addition fails to thicken with age [6].

The basement membrane of the ciliary epithelium is, in the present study, found to thicken not only in diabetic man, but also in experimentally diabetic animals. The thickening is evidently initiated as a result of diabetes, and can be inhibited by careful control of the metabolic symptoms of diabetes with insulin. In this respect, the epithelial basement membrane evidently behaves like that of blood vessels, particularly capillaries of the retina, renal glomerulus, and skeletal muscle [2].

There is reason to speculate that basement membrane thickening in capillaries might be elicited by increased blood pressure [11], and indeed thickened basement membrane has been described in arterioles, capillaries, and venules of experimentally hypertensive animals [12]. In order to learn whether or not epithelial basement membrane may vary with blood pressure, we included in our study tissue obtained post mortem from non-diabetic patients who had been diagnosed as hypertensive $2-8$ years before death. Epithelial basement membrane in this group appeared to be normal. The results suggest that the thickening we observed in diabetic subjects is not commonplace among ill subjects, and may be unrelated to the presence or absence of elevated blood pressure.

The thickness of the ciliary epithelial basement membrane in man has been shown to increase with age as well as with the presence of diabetes [4]. The human and animal populations we examined were small and of relatively uniform ages, and under these circumstances no correlation with age was evident. One reason we chose to measure this basement membrane is that in normal man it is several $\mu \mathrm{m}$ thick, and readily measurable by light microscopy. We were dismayed to find that in normal dogs this basement membrane is $<1 \mu \mathrm{m}$ thick, and is accordingly difficult to measure by light 
microscopy. However, 5 years of chronic hyperglycaemia and glucosuria in dogs proved sufficient nearly to double its thickness, as seen by comparison of either the minimum thickness or random measurements.

The basement membrane of the lens epithelium, i.e. anterior lens capsule, has not previously been shown to thicken during diabetes, but has been examined quantitatively in only a single study. In that study, rats in which body growth had been markedly inhibited as a result of streptozotocin diabetes were found to have lenses lighter in weight and with thinner capsules than those of normal rats [13]. A failure of normal lens growth in those rats can perhaps account for the thinness of the lens capsule. In the present study, the lens capsule was abnormally thick after 5 years of poorly controlled diabetes in dogs, and showed a significant correlation with duration of diabetes in man, a correlation greater than can be attributed to aging. Thickening of the lens capsule in the diabetic dog was prevented by better diabetic control, and seemed associated with the presence of cataract and chronic hyperglycaemia. Even in the absence of diabetes, lens disorders sufficient to produce cataract can result in thickening of the capsule [14]. No suitable data were available by which to judge diabetic control in the human subjects.

Various mechanisms have been postulated to account for thickening of basement membrane in diabetes. An increased synthesis of basement membrane material appears to be involved, but other mechanisms, including a possible decrease in its degradation or removal, have not been ruled out $[11,15]$. In at least some tissues, basement membrane is believed to accumulate when cells proliferate in response to injury or normal aging $[16,17]$. Vracko postulated that the basement membrane thickening typical of diabetes may similarly be a result of accelerated cell turnover [17].

In contrast to the basement membrane thickening that occurs in numerous tissues in diabetes, the basement membrane of the corneal mesothelium shows no tendency whatsoever to thicken during diabetes. We chose to examine the corneal mesothelium in particular because in man its response to injury is known to be quite unlike that of blood vessel endothelium or the ciliary or lens epithelium. Repair of the corneal mesothelium in man [18] and dogs [19] is accomplished chiefly by enlargement and migration of existing cells rather than by cell division. It seems that the notoriously low regenerative activity of these cells might account in part for the finding that although the basement membranes of the lens, ciliary epithelium, and ocular blood vessels tend to thicken during diabetes, the basement membrane of the corneal mesothelium nearby does not.

Acknowledgements. We are indebted to Dr. G. de Venecia for advice and assistance with the review of human autopsy eyes. The cooperation of Dr. J.M.B. Bloodworth Jr, with whom diabetic animals were maintained for a number of years, is especially appreciated. The tech- nical skills of $\mathrm{M}$. Larson and S. Smith are gratefully acknowledged. This work was supported in part by Public Health Service research grant EY00300 from the National Eye Institute.

\section{References}

1. Osterby R (1975) Early phases in the development of diabetic glomerulopathy. Acta Med Scand (Suppl) 574

2. Bloodworth JMB, Engerman RL (1980) Experimental diabetic glomerulosclerosis. In: Podolsky S, Viswanathan M (eds) Secondary Diabetes. Raven Press, New York, pp 521-540

3. Rasch R (1979) Prevention of diabetic glomerulopathy in streptozotocin diabetic rats by insulin treatment. Glomerular basement membrane thickness. Diabetologia 16: 319-324

4. Yamashita T, Becker B (1961) The basement membrane in the human diabetic eye. Diabetes 10: 167-174

5. Osterby-Hansen R, Lundbaek K (1970) The basement membrane morphology in diabetes mellitus. In: Ellenberg M, Rifkin H (eds) Diabetes mellitus theory and practice. McGraw Hill, New York, pp 178-209

6. Vracko R (1978) Effects of aging and diabetes on basal lamina thickness of six cell types. In: Kefalides $N$ (ed) Biology and Chemistry of Basement Membranes. Academic Press, New York, pp 483-493

7. Engerman R, Bloodworth JMB, Nelson S (1977) Relationship of microvascular disease in diabetes to metabolic control. Diabetes 26: 760-769

8. Bischoff A (1968) Diabetische Neuropathie. Dtsch Med Wochenschr 93: 237-241

9. Johnson PC, Brendel K, Meezan E (1981) Human diabetic perineurial cell basement membrane thickening. Lab Invest 44: 265-270

10. Taylor HR, Kimsey RA (1981) Corneal epithelial basement membrane changes in diabetes. Invest Ophthalmol Vis Sci 20: 548-553

11. Williamson JR, Kilo C (1977) Current status of capillary basement membrane disease in diabetes mellitus. Diabetes 26: 65-73

12. Sekino T (1977) Electron microscopic studies on the retinal vessels of spontaneously hypertensive rats (SHR). Jpn J Ophthalmol 21: 192-205

13. Fisher RF (1979) Factors which influence the thickness of basement membrane in diabetes. Trans Ophthalmol Soc UK 99: 10-12

14. Fukui S, Yamashima I (1978) Biochemical alterations of the lens capsule in mice with hereditary cataract. Exp Eye Res 26: 499-506

15. Brownlee M, Spiro R (1979) Glomerular basement membrane metabolism in the diabetic rat. Diabetes 28: 121-125

16. Pierce GB, Nakane PK (1972) Basement membrane: synthesis and role in disease. In: Takeuchi T, Ogawa K, Fujita S (eds) Proceedings of the International Congress of Histochemistry and Cytochemistry, Japan Society of Histochemistry and Cytochemistry, Kyoto, pp 125-126

17. Vracko R (1974) Basal lamina layering in diabetes mellitus. Diabetes 23:94-104

18. Waring GO, Laibson PR, Rodrigues M (1974) Clinical and pathologic alterations of Descemet's membrane. Surv Ophthalmol 18: 325-368

19. Gwin RM, Lerner I, Warren JK, Gum G (1982) Decrease in canine corneal endothelial cell density and increase in corneal thickness as functions of age. Invest Ophthalmol Vis Sci 22: 267-271

Received: 13 August 1981

and in revised form: 5 April 1982

Dr. R.L. Engerman

Department of Ophthalmology

University of Wisconsin

600 Highland Avenue

Madison, Wisconsin 53792, USA 